\title{
TRADUÇÃO / TRANSLATION
}

\section{CARTA DE DESCARTES A ELISABETH}

\author{
Tradução e Introdução de Carlos Arthur R. do NASCIMENTO*
}

\section{INTRODUÇÃO}

Já se observou que "entre aqueles a quem se dá o nome de "grandes" da filosofia... só Descartes não escreveu um texto explicitamente político”' (1, p. 29). Se ressalvarmos alguns trechos do Discurso do método em que a política comparece muito mais para ser excluida das preocupações do filósofo do que para se constituir em objeto de reflexão, só a podemos encontrar na obra cartesiana publicada através de "desvios"**. No entanto, malgrado ele mesmo, Descartes é levado a falar de política em suas cartas. Notadamente num grupo de cartas à sua estimada princesa Elisabeth***. Apresentamos aqui a tradução de uma destas cartas, notável sobretudo porque nela se desenvolve um comentário de $O$ príncipe de Maquiavel e onde se percebe que, mesmo quando Descartes se inclina a adotar os pontos de vista do florentino, o faz por motivos inteiramente distintos.

Utilizamos o texto da edição Adam et Tannery (2) que acompanha esta tradução. $\mathrm{Na}$ transcrição do original a ortografia francesa foi atualizada, utilizando para tanto o texto da Bibliothèque de la Pléiade (3).

\section{Descartes à Elisabeth}

Egmond, septembre 1646

Madame,

J'ai lu le livre dont Votre Altesse m'a commandé de lui écrire mon opinion, et j'y trouve plusieurs préceptes qui me semblent fort bons; comme entre autres au 19 et 20e chapitres: Qu'un Prince doit toujours éviter la haine et le mépris de ses sujets, et que l'amour du peuple vaut mieux que les forteresses. Mais il y en a aussi plusieurs autres que je ne saurais approuver. Et je crois que ce en quoi l'Auteur a le plus manqué, est qu'il n'a pas mis assez de distinction entre les Princes qui ont acquis un Etat par des

\footnotetext{
* Departamento de Filosofia - Faculdade de Comunicação e Filosofia - Pontifícia Universidade Catolica - 01000 SP.

** A expressão é de Châtelet (1, p. 29). É o que tentaria, por exemplo, A. Negri (4).

*** Ver a respeito (5) e (6).
} 
DESCARTES, R. - Cart a de Descartes, Egmond, septembre 1646, para Elisabeth. Trad. ( arlos Arthur R. do Nascimento. Trans/Form/Acão, São Paulo, 7:61-68, 1984.

voies justes, et ceux qui l'ont usurpé par des moyens illégitimes; et qu'il a donné à tous, généralement, les préceptes qui ne sont propres qu'à ces derniers. Car comme, en bâtissant une maison dont les fondements sont si mauvais qu'ils ne sauraient soutenir des murailles hautes et épaisses, on est obligé de les faire faibles et basses, ainsi ceux qui ont commencé à s'établir par des crimes sont ordinairement contraints de continuer à commettre des crimes, et ne se pourraient maintenir s'ils voulaient être vertueux.

C'est au regard de tels Princes qu'il a pu dire, au chapitre 3: Qu'ils ne sauraient manquer d'être hais de plusieurs; et qu'ils ont souvent plus d'avantage à faire beaucoup de mal qu'à en faire moins, pour ce que les légère offenses suffisent pour donner la volonté de se venger, et que les grandes en ôtent le pouvoir. Puis, au chapitre 15: Que, s'ils voulaient être gens de bien, il serait impossible qu'ils ne se ruinassent parmi le grand nombre de méchants qu'on trouve partout. Et au chapitre 19: Qu'on peut être hai pour de bonnes actions aussi bien que pour de mauvaises.

Sur lesquels fondements il appuie des préceptes très tyranniques, comme de vouloir qu'on ruine tout un pays, afin d'en demeurer le maître; qu'on exerce de grandes cruautés, pourvu que ce soit promptement et tout à la fois; qu'on tâche de paraître homme de bien, mais qu'on ne le soit pas véritablement; qu'on ne tienne sa parole qu'aussi longtemps qu'elle sera utile; qu'on dissimule, qu'on trahisse; et enfin que, pour régner, on se dépouille de toute humanité, et qu'on devienne le plus farouche de tous les animaux.

Mais c'est un très mauvais sujet pour faire des livres, que d'entreprendre d'y donner de tels préceptes, qui, au bout du compte, ne sauraient assurer ceux auxquels il les donne; car, comme il avoue lui-même, ils ne se peuvent garder du premier qui voudra négliger sa vie pour se venger d'eu. Au lieu que, pour instruire un bon Prince, quoique nouvellement entré dans un Etat, il me semble qu'on lui doit proposer des maximes toutes contraires, et supposer que les moyens dont il s'est servi pour s'établir ont été justes; comme, en effet, je crois qu'ils le sont presque tous, lorsque les Princes qui les pratiquent les estiment tels; car la justice entre les Souverains a d'autres limites qu'entre les particuliers, et il semble qu'en ces recontres Dieu donne le droit à ceux auxquels il donne la force. Mais les plus justes actions deviennent injustes, quand ceux qui les font les pensent telles.

On doit aussi distinguer entre les sujets, les amis ou alliés et les ennemis. Car, au regard de ces derniers, on a quasi permission de tout faire, pourvu qu'on en tire quelque avantage pour soi ou pour ses sujets; et je ne désaprouve pas, en cette occasion, qu'on accouple le renard avec le lion et qu'on joigne l'artifice à la force. Même je comprends, sous le nom d'ennemis, tous ceux qui ne sont point amis ou alliés, pour ce qu'on a droit de leur faire la guerre, quand on y trouve son avantage, et que, commençant à devenir suspects et redoutables, on a lieu de s'en défier. Mais j'excepte une espèce de tromperie, qui est si directement contraire à la société, que je ne crois pas qu'il soit jamais permis de s'en servir, bien que notre Auteur l'approuve en divers endroits, et qu'elle ne soit que trop en pratique: c'est de feindre d'être ami de ceux qu'on veut perdre, afin de les pouvoir mieux surprendre. L'amitié est une chose trop sainte pour en abuser de la sorte; et celui qui aura pû feindre d'aimer quelqu'un, pour le trahir, mérite que ceux qu'il voudra par après aimer véritablement, n'en croient rien et le haissent.

Pour ce qui regarde les alliés, un Prince leur doit tenir exactement sa parole, même lorsque cela lui est préjudiciable; car il ne le saurait être tant, que la réputation de ne manquer point à faire ce qu'il a promis lui est utile; et il ne peut acquérir cette réputation que par de telles occasions, où il y va pour lui de quelque perte; mais en celles qui le ruineraient tout à fait, le droit des gens le dispense de sa promesse. Il doit aussi user de 


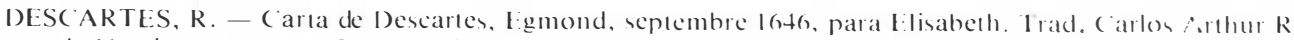
do Nascimento. Trans/Form/Acào, São Paulo, 7:61-68, 1984t

beaucoup de circonspection, avant que de promettre, afin de pouvoir toujours garder sa foi. Et bien qu'il soit bon d'avoir amitié avec la plupart de ses voisins, je crois néanmoins que le meilleur est de n'avoir point d'étroites alliances, qu'avec ceux qui sont moins puissants. Car, quelque fidélité qu'on se propose d'avoir, on ne doit pas attendre la pareille des autres, mais faire son compte qu'on en sera trompé, toutes les fois qu'ils y trouveront leur avantage; et ceux qui sont plus puissants l'y peuvent trouver, quand ils veulent, mais non pas ceux qui le sont moins.

Pour ce qui est des sujets, il y en a de deux sortes: à savoir les grands et le peuple. Je comprends, sous le nom de grands, tous ceux qui peuvent former des partis contre le Prince, de la fidélité desquels il doit être très assure; ou, s'il ne l'est pas, tous les politiques sont d'accords qu'il doit employer tous ses soins à les abaisser, et qu'en tant qu'ils sont enclins à brouiller l'Etat, il ne les doit considérer que comme ennemis. Mais, pour ses autres sujets, il doit surtout éviter leur haine et leur mépris; ce que je crois qu'il peut toujours faire, pourvu qu'il observe exactement la justice à leur mode (c'est-à-dire suivant les lois auxquelles ils sont accoutumés), sans être trop rigoureux aux punitions, ni trop indulgent aux grâces, et qu'il ne remette pas de tout à ses Ministres, mais que, leur laissant seulement la charge des condamnations plus odieuses, il témoigne avoir lui-même le soin de tout le reste; puis aussi, qu'il retienne tellement sa dignité, qu'il ne quitte rien des honneurs et des déférences que le peuple croit lui être dues, mais qu'il n'en demande point davantage, et qu'il ne fasse paraître en public que ses plus sérieuses actions, ou celles qui peuvent être approuvées de tous, réservant à prendre ses plaisirs en particulier, sans que ce soit jamais au dépens de personne; et enfin qu'il soit immuable et inflexible, non pas aux premiers desseins qu'il aura formés en soi-même, car d'autant qu'il ne peut avoir l'oeil partout, il est nécessaire qu'il demande conseil, el entende les raisons de plusieurs, avant que de se résourdre; mais qu'il soit inflexible touchant les choses qu'il aura témoigné avoir résolues, encore même qu'elles lui fussent nuisibles; car malaisément le peuvent-elles être tant que serait la réputation d'être léger et variable.

Ainsi je désapprouve la maxime du chapitre 15: Que, le monde étant fort corrompu, il est impossible qu'on ne se ruine, si l'on veut être toujours homme de bien; et qu'un Prince, pour se maintenir, doit apprendre à être méchant, lorsque l'occasion le requiert; si ce n'est peut-être que, par un homme de bien, il entende un homme superstitieux et simple, qui n'ose donner bataille au jour du Sabbat, et dont la conscience ne puisse être en repos, s'il ne change la religion de son peuple. Mais, pensant qu'un homme de bien est celui qui fait tout ce que lui dicte la vraie raison, il est certain que le meilleur est de tâcher à l'être toujours.

Je ne crois pas aussi ce qui est au chapitre 19: Qu'on peut autant être hai pour les bonnes actions, que pour les mauvaises, sinon en tant que l'envie est une espèce de haine; mais cela n'est pas le sens de l'Auteur. Et les Princes n'ont pas coutume d'être enviés par le commum de leurs sujets; ils le sont seulement par les grands, ou par lcurs voisins, auxquels les mêmes vertus qui leur donnent de l'envie, leur donnent aussi de la crainte; c'est pourquoi jamais on ne doit s'abstenir de bien faire, pour éviter cette sorte de haine; et il n'y en a point qui leur puisse nuire, que celle qui vient de l'injustice ou de l'arrogance que le peuple juge être en eux. Car on voit même que ceux qui ont été condamnés à la mort, n'ont point coutume de haïr leurs juges, quand ils pensent l'avoir méritée; et on souffre aussi avec patience les maux qu'on n'a point mérités, quand on croit que le Prince, de qui on les reçoit, est en quelque façon contraint de les faire, et qu'il en a du déplaisir pour ce qu'on estime qu'il est just qu'il préfère l'utilité publique à celle des particuliers. Il y a seulement de la difficulté, lorsqu'on est obligé de satisfaire à deux partis qui jugent différemment de ce qui est juste, comme lorsque les Empereurs 
Romains avaient à contenter les Citoyens et les Soldats; auquel cas il est raisonnable d'accorder quelque chose aux uns et aux autres, et on ne doit pas entreprendre de faire venir tout d'un coup à la raison ceux qui ne sont pas accoutumés de l'entendre; mais il faut tâcher peu-à-peu, soit par des écrits publics, soit par les voix des Prédicateurs, soit par tels autres moyens, à la leur faire concevoir. Car enfin le peuple souffre tout ce qu'on lui peut persuader être juste, et s'offense de tout ce qu'il imagine d'être injuste; et l'arrogance des Princes, c'est-à-dire l'usurpation de quelque autorité, de quelques droits, ou de quelques honneurs qu'il croit ne leur être point dus, ne lui est odieuse, que pour ce qu'il la considère comme une espèce d'injustice.

Au reste, je ne suis pas aussi de l'opinion de cet Auteur, en ce qu'il dit en sa Préface: Que, comme il faut être dans la plaine, pour mieux voir la figure des montagnes, lorsqu'on en veut tirer le crayon ainsi on doit être de condition privée, pour bien connaitre l'office d'un Prince. Car le crayon ne représente que les choses qui se voient de loin; mais les principaux motifs des actions des Princes sont souvent des circonstances si particulières que, si ce n'est qu'on soit Prince soi-même, ou bien qu'on ait été fort longtemps participant de leurs secrets, on ne les saurait imaginer.

C'est pourquoi je mériterais d'être moqué, si je pensais pouvoir enseigner quelque chose à Votre Altesse en cette matière; aussi n'est-ce pas mon dessein, mais seulement de faire que mes lettres lui donnent quelque sorte de divertissement, qui soit différent de ceux que je m'imagine qu'elle a en son voyage, lequel je lui souhaite parfaitement heureux: comme sans doute il le sera, si Votre Altesse se résout de pratiquer ces maximes qui enseignent que la félicité d'un chacun dépend de lui-même, et qu'il faut tellement se tenir hors de l'empire de la Fortune, que, bien qu'on ne perde pas les occasions de retenir les avantages qu'elle peut donner, on ne pense pas toutefois être maheureux lorsqu'elle les refuse; et pour ce qu'en toutes les affaires du monde il y a quantité de raisons pour et contre, qu'on s'arrête principalement à considérer celles qui servent à faire qu'on approuve les choses qu'on voit arriver. Tout ce que j'estime le plus inévitable sont les maladies du corps, desquelles je prie Dieu qu'il vous préserve, et je suis avec toute la dévotion que je puis avoir, etc... 
DESCARTES, R. - Carta de Descartes, Egmond, septembre 1646, para Elisabeth. Trad. Carlos Arthur R. do Nascimento. Trans/Form/Ação, São Paulo, 7:61-68, 1984.

\section{REFERÊNCIAS BIBLIOGRÁFICAS}

1. CHÂTELET, F. - A questão da história dä filosofia hoje. In:_ Politicas da filosofia. Lisboa, Moraes, 1977. p.23-42.

2. DESCARTES, R. - Oeuvres, Publièes par Charles Adan et Paul Tannery. Correspondence, Juillet 1643 - Avril 1647. Paris, Léopold Cerf. 1901, Tome 4, 485-494.

3. DESCARTES, R. - Oeuvres et Lettres. Paris, Pléiade, Gallimard, 1953. p. 1236-1241.
4. NEGRI, A. - Descartes politico. $\mathrm{O}^{-}$della ragionevole ideologia. Milano, Feltrinelli, 1970.

5. POLIN, R. - Descartes et la philosophie politique. In: - L'Aventure de l'esprit, Mélanges Alexandre Koyré II. Paris, Hermann, 1964. p. 381-399.

6. REGNAULT, F. - La pensée di prévu (Descartes et Machiavel) e Descartes et Elisabeth (Quatres lettres sur Machiavel). In: Cahiers pour I 'analyse 6 (La politique des philosophes). $\mathrm{Pa}$ ris, Seuil, p. 21-62. 